\title{
EP4 Antagonist ONO-4578
}

National Cancer Institute

\section{Source}

National Cancer Institute. EP4 Antagonist ONO-4578. NCI Thesaurus. Code C150376.

An orally bioavailable antag onist of the prostaglandin E2 receptor subtype 4 (PT GER4; EP4), with potential analgesic, immunomodulating and antineoplastic activities. Upon administration, the EP4 antagonist ONO-4578 selectively targets and binds to EP4, inhibiting the binding of the immunosuppressive prostaglandin E2 (PGE2) to EP4. This prevents the activation of EP4 and inhibits PGE2-EP4-mediated signaling, thereby inhibiting proliferation of tumor cells in which the PGE2-EP4 signaling pathway is overactivated. In addition, EP4 inhibition prevents the activity of tumor-associated myeloid cells (TAMCs) in the tumor microenvironment (TME) by inhibiting interleukin-23 (IL-23) production and the IL-23-mediated expansion of Th17 cells. EP4, a prostanoid receptor, is a G protein-coupled receptor that is expressed in certain types of cancers; it promotes tumor cell proliferation and invasion. 